DOI: https://doi.org/10,11144/Javeriana.umed58-4.dmmm

\title{
Guía de práctica clínica para el tratamiento de la diabetes mellitus tipo 2: metas de manejo
}

\section{Clinical Practice Guideline for Treatment of type 2 Diabetes Mellitus: Therapeutic Targets}

\author{
Pablo Aschner M. \\ Hospital Universitario San Ignacio, Colombia \\ Óscar Mauricio Muñoz V.a \\ Hospital Universitario San Ignacio, Colombia \\ Diana Girón C. \\ Pontificia Universidad Javeriana, Colombia \\ Olga Milena García M. \\ Hospital Universitario San Ignacio, Colombia \\ Daniel Gerardo Fernández Á. \\ Hospital Universitario San Ignacio, Colombia \\ Luz Ángela Casas \\ Universidad del Valle, Colombia \\ Luisa Fernanda Bohórquez \\ Universidad Nacional de Colombia, Colombia \\ Clara María Arango T. \\ Universidad de Antioquia, Colombia
}

Fecha de recepción: 27/09/2016 | Fecha de aprobación: 18/01/2017

\author{
Liliana Carvajal \\ Asociación Colombiana de Diabetes, Colombia \\ Doris Amanda Ramírez \\ Universidad Nacional de Colombia, Colombia \\ Juan Guillermo Sarmiento \\ Hospital Universitario San Ignacio, Colombia \\ Cristian Alejandro Colon \\ Hospital Universitario San Ignacio, Colombia \\ Néstor Fabián Correa G. \\ Pontificia Universidad Javeriana, Colombia \\ Pilar Alarcón R. \\ Pontificia Universidad Javeriana, Colombia \\ Álvaro Andrés Bustamante S. \\ Pontificia Universidad Javeriana, Colombia \\ Víctor Fino* \\ ERnesto Penagos**
}

\footnotetext{
aAutor de correspondencia. Correo electrónico: o.munoz@javeriana.edu.co

* Representante de la población blanco.

** Representante de la población blanco.
}

Cómo citar: Aschner P, Muñoz OM, Girón D, García OM, Fernández DG, Casas LA, Bohórquez LF, Arango CM, Carvajal L, Ramírez DA, Sarmiento JG, Colón CA, Correa NF, Alarcón P, Bustamante ÁA, Fino V, Penagos E. Guía de práctica clínica para el tratamiento de la diabetes mellitus tipo 2: metas de manejo. Univ Med. 2017;58(4): 1-9. doi: https://doi.org/10.1 1144/Javeriana.umed58-4.dmmm

\section{RESUMEN}

Introducción: Está demostrado que alcanzar y mantener una hemoglobina glicosilada $(\mathrm{HbA} 1 \mathrm{c})$ del $7 \%$ reduce la incidencia de complicaciones micro y macrovasculares en adultos jóvenes con diabetes 
mellitus tipo 2 (DMT2). Se ha propuesto que esta meta de manejo debe individualizarse en pacientes mayores de 65 años de edad o en aquellos con antecedente de enfermedad cardiovascular; sin embargo, la evidencia es discutida. Objetivos: Definir los potenciales riesgos y beneficios de la terapia intensificada para DMT2 buscando valores de $\mathrm{HbA1}<7 \%$ en pacientes mayores de 65 años, con comorbilidades significativas y sin estas, y en grupos de pacientes con eventos cardiovasculares previos. Métodos: Se elaboró la guía de práctica clínica, siguiendo los lineamientos de la guía metodológica del Ministerio de Salud y Protección Social colombiano. Se revisó la evidencia disponible de forma sistemática y se formularon las recomendaciones utilizando la metodología GRADE. Conclusiones: En los pacientes con DMT2 mayores de 65 años y en aquellos con antecedente de enfermedad cardiovascular, se recomienda no intensificar el manejo para alcanzar valores de $\mathrm{HbA1c}$ cercanos a lo normal (<6,5\%). Las metas de manejo en el grupo de mayores de 65 años deberán definirse para cada paciente de forma individual de acuerdo con una valoración integral que tenga en cuenta su riesgo de hipoglucemia y pronóstico vital.

Palabras clave

diabetes mellitus tipo 2; metas; guías de práctica clínica.

\begin{abstract}
Introduction: It is demonstrated that reach and maintain a glycated hemoglobin $(\mathrm{HbA} 1 \mathrm{c})$ of $7 \%$ reduce the incidence of macro and micro vascular complications in young adults with type 2 diabetes. It has been proposed that this therapeutic goal must be individualized in patients older than 65 years or in those with previous cardiovascular disease, however the evidence supporting this adjustment is controversial. Aim: To define the risk and benefits of intensified therapy to achieve $\mathrm{HbA} 1<7 \%$ in DMT2 patients older than 65 years, with or without significant comorbidities, and in patients with previous cardiovascular events. Methods: A clinical practice guide has been developed following the broad outline of the methodological guide from the Colombian Ministry of Health and Social Welfare, with the aim of systematically gathering scientific evidence and formulating recommendations using the GRADE methodology. Conclusions: In patients with DMT2 who are older than 65 years of age, and in those with antecedents of cardiovascular disease it is recommended not to intensify treatment to reach $\mathrm{HbA} 1 \mathrm{c}$ values close to normal $(<6,5 \% \mathrm{HbA} 1 \mathrm{c})$. The therapeutic goals in patients older than 65 years should be individualized according with an integral evaluation considering hypoglycemia risk and life-threatening situation.
\end{abstract}

Keywords

type 2 diabetes mellitus; goals; clinical practice guidelines.

\section{Introducción}

El control de la diabetes mellitus tipo 2 (DMT2) debe ser temprano, efectivo y sostenido para prevenir las complicaciones crónicas y evitar el efecto deletéreo de la memoria metabólica [1]. Los estudios clínicos controlados y aleatorizados como el UK Prospective Diabetes Study (UKPDS) han demostrado que si la DMT2 se trata adecuadamente desde un comienzo, es posible reducir la incidencia de complicaciones crónicas atribuibles a la hiperglucemia prolongada $[2,3]$. En particular, se ha demostrado un disminución en la incidencia de retinopatía, nefropatía y neuropatía al cabo de diez años de una estrategia de manejo intensivo, y con un seguimiento más largo, de hasta veinte años, se demostró también una reducción de eventos cardiovasculares fatales y no fatales [4].

A la hora de definir las metas de manejo, los estudios clínicos controlados a largo plazo y su posterior seguimiento han demostrado que alcanzar y mantener una hemoglobina glicosilada (HbA1c) promedio del $7 \%$ reduce la incidencia de complicaciones micro y macrovasculares de la DMT2. Adicionalmente, estudios diseñados para evaluar si la intensificación del tratamiento para alcanzar una HbA1c normal $(<6,5$ $\%)$ no han encontrado que una terapia más agresiva produzca beneficios que superen el riesgo de efectos adversos; incluso se ha presentado mayor mortalidad y deterioro en la calidad de vida [5,6,7]. Así, en pacientes con DMT2 sin comorbilidades serias y con edad $\leq 65$ años, las guías internacionales sugieren disminuir las concentraciones de $\mathrm{HbAlc}$ hasta en un $6,5 \%$, especialmente si ya tienen indicios de microangiopatía. Cabe, sin embargo, recalcar que las metas deben ser individualizadas, teniendo en cuenta las características de los pacientes y su grado de vulnerabilidad, en especial la hipoglucemia [8].

Múltiples autores han propuesto que en los mayores de 65 años de edad los objetivos del tratamiento deberían cambiar, teniendo en cuenta su menor expectativa de vida, la probabilidad de generar mayor frecuencia de efectos adversos potencialmente graves, además 
de poder empeorar sus comorbilidades. En este grupo etario se hace indispensable una valoración integral periódica que determine de forma individual la situación funcional, social, clínica y mental $[9,10]$. Dicha valoración permitiría tomar decisiones con miras a minimizar los efectos adversos del tratamiento que puedan deteriorar la calidad de vida, como lo es la hipoglucemia.

Según lo mencionado, los consensos internacionales han acordado diferenciar dos grupos de ancianos, con características diferenciales y, por tanto, objetivos de $\mathrm{HbA1c}$ diferenciales [9]. En un primer grupo se incluirían pacientes ancianos sin complicaciones, es decir, con buena situación funcional, adecuada situación cognitiva y una buena expectativa de vida, quienes podrían beneficiarse de un control glucémico más estricto. Un segundo grupo incluye pacientes ancianos frágiles, es decir, aquellos con expectativa de vida corta, múltiples comorbilidades u alto riesgo de hipoglucemia, en quienes resultaría aceptable perseguir objetivos HbA1c más laxos.

Igualmente, se ha planteado que debería individualizarse la meta de manejo en pacientes que ya han presentado eventos cardiovasculares, teniendo en cuenta que las personas con DMT2 tienen el doble de riesgo de presentar enfermedad cardiovascular cuando se comparan con pacientes sin diabetes, incluso después de excluir otros factores de riesgo [11]. Una meta de $\mathrm{HbA1c}$ del $7 \%$ es aceptada para reducir el riesgo de enfermedad microvascular; sin embargo, una meta de $\mathrm{HbA1c}$ con miras a minimizar el riesgo de enfermedad macrovascular está menos establecida [12]. Se han propuesto, por ejemplo, que concentraciones del 6,0\%-6,5\% podrían estar indicadas en pacientes jóvenes de alto riesgo cardiovascular, individualizando el manejo para garantizar que no se presenten hipoglucemia u otros efectos adversos asociados. Sin embargo, la evidencia al respecto no es contundente y algunos estudios han demostrado que no todos los pacientes con enfermedad cardiovascular se benefician de manejo agresivo de la glucemia [13].

El Ministerio de Salud y Protección Social encargó a la Pontificia Universidad Javeriana y a la Alianza del Centro Nacional de Investigación en Evidencia y Tecnologías en Salud (Cinets), conformada adicionalmente por la Universidad Nacional de Colombia y la Universidad de Antioquia, el desarrollo de una Guía clínica sobre la prevención, detección temprana, diagnóstico, tratamiento y seguimiento de la diabetes mellitus en la población mayor de 18 años. El resultado de ese proceso, en lo referente a las metas de manejo, incluyendo grupos especiales como mayores de 65 años y pacientes con eventos cardiovasculares previos, se presenta de forma resumida en el presente artículo. Documentos complementarios abordarán los temas de tratamiento inicial y manejo de pacientes que fallaron a la terapia de primera línea.

Cabe resaltar que en este proceso también participaron activamente la Asociación Colombiana de Endocrinología, así como la Asociación Colombiana de Diabetes, y el Cinets.

\section{Métodos}

El proceso de desarrollo de la guía se describe detalladamente en el Manual para desarrollo de guías de práctica clínica y en la actualización de dicha metodología (disponible en la página web del Ministerio de Salud y Protección Social: http ://gpc.minsalud.gov.co) [14].

El desarrollo de la guía partió de preguntas específicas seleccionadas por su importancia clínica y se dio prioridad a los temas en que se consideró aún existe una discusión activa en la literatura mundial y a aquellos temas en los que se detectó una variabilidad injustificada en las conductas realizadas por los profesionales de salud en Colombia. Cabe aclarar que se aceptaron como premisas a la presente guía aquellos temas ampliamente aceptados en la literatura mundial y que no ameritan, por tanto, un nuevo análisis de la literatura científica, y se presentan en el presente documento.

La metodología planteada garantiza una búsqueda sistemática de la evidencia científica (incluyendo tanto revisiones sistemáticas de la literatura como estudios primarios). Así mismo, establece de forma clara los criterios para 
seleccionar la evidencia que se va a utilizar, evaluando cuidadosamente su calidad. La versión completa de la guía, en la que se presentan los resultados de todas estas evaluaciones, puede consultarse en la página web del Ministerio de Salud y Protección Social colombianos, así como en la página web de la Alianza Cinets.

El grupo metodológico preparó un resumen de la evidencia disponible y lo presentó al panel completo durante las reuniones de generación de recomendaciones. Este panel incluyó a representantes de diversas especialidades médicas (endocrinólogos, médicos internistas, médicos de familia y médicos generales), así como a otros profesionales de la salud (nutricionistas y terapeutas físicos). Cabe resaltar que durante todo el proceso se contó también con representantes de los pacientes, que aportaron activamente en la generación de recomendaciones. Todos los integrantes del panel presentaron de modo abierto la declaración de sus conflictos de interés. Estos documentos están disponibles en la versión completa de la guía.

Durante las reuniones de generación de recomendaciones se siguió la metodología propuesta por el Grading of Recommendations Assessment, Development and Evaluation Working Group (GRADE), que permite tener en cuenta no solo la calidad de la evidencia disponible, sino consideraciones de costos, preferencias de los pacientes y relación entre los beneficios y los riesgos de las tecnologías de interés (pruebas, estrategias de manejo, intervenciones o medicamentos). Las recomendaciones presentadas muestran, por tanto, la fuerza de la recomendación (fuerte o débil), su dirección (a favor o en contra de la intervención), así como la calidad de la evidencia que la respalda (muy baja, baja, moderada, alta o consenso de expertos).

La versión final de la guía fue evaluada por pares nacionales, seleccionados por el Ministerio de Salud y de la Protección Social colombiano, expertos tanto en DMT2 como en metodología. Sus aportes fueron tenidos en cuenta por el grupo desarrollador de la guía.

\section{Resultados}

Múltiples revisiones sistemáticas $[15,16,17,18,19,20]$ han evaluado el impacto del tratamiento intensificado de la DMT2 (metas de HbA1c menores al $7 \%$ ) comparado con manejo convencional. Si bien ninguna de ellas evaluó exclusivamente pacientes mayores de 65 años de edad, los datos en ellas incluidos, y algunos subanálisis de experimentos clínicos recientes [21,22], permiten analizar la evidencia disponible actualmente para esta población. En resumen, la información disponible es la siguiente:

El tratamiento intensificado enfocado en obtener como meta de manejo una HbA1c cercana al valor normal no impacta el riesgo de mortalidad global, ni el riesgo de mortalidad de origen cardiovascular, tanto en la población general como en el subgrupo de mayores de 60 años, aunque se observó heterogeneidad en este último grupo (RR: 1,13; IC95 \%: 0,81-1,57; $\mathrm{I}^{2}=$ $72 \%)[16]$.

En un estudio para evaluar el beneficio del control glucémico intensivo ( $\mathrm{HbA} 1 \mathrm{c}<6 \%$ ) sobre el riesgo cardiovascular que tuvo que ser suspendido por un aumento de la mortalidad en el grupo de control intensivo, el análisis de subgrupos demostró que dicho aumento fue significativo en menores de 65 años; pero no en los que tenían mayor edad (HR: 0,97; IC95 \%: 0,7-1,36 para mortalidad cardiovascular y HR: 1,06; IC95 \%: 0,84-1,34 para muerte por cualquier causa). Por el contrario, la disminución en la incidencia de infarto del miocardio no fatal se observó solo en el subgrupo de menores de 65 años en ese mismo análisis. La incidencia de hipoglucemia que requirió asistencia médica fue casi el doble en los que tenían 65 o más años (incidencia anual 4,45 \% vs. 2,45\%) y, de hecho, la hipoglucemia fue tan frecuente al inicio del estudio en mayores de 80 años que la inclusión de pacientes en este grupo etario se suspendió [21].

El tratamiento enfocado en obtener como meta una HbA1c cercana al valor normal no impacta el riesgo de ataque cerebrovascular en la población general. Hallazgos similares se encontraron en el subanálisis teniendo en cuenta 
exclusivamente mayores de 62 años (RR: 1,01; IC95 \%: 0,91-1,11) [20]

No existen datos específicos a calidad de vida para mayores de 65 años; sin embargo, datos obtenidos en la población general sugieren que no hay impacto del manejo intensificado en las mediciones de estado general de salud, síntomas asociados a la diabetes o depresión.

Una revisión sistemática reciente con una muestra grande $(34,912$ pacientes DMT2 con edades de hasta 72 años) no logró hacer el análisis planeado para el grupo $\geq 65$ años; pero encontró que, en general, el control glucémico intensivo reduce significativamente la incidencia de infarto del miocardio no fatal (RR: 0,87; IC95 \%: 0,77-0,98) y de amputaciones (RR: 0,45; IC95 \%: 0,45-0,94) con calidad moderada de la evidencia y aumenta el riesgo de hipoglucemia (RR: 2,18; IC95 \%: 1,53-3,11) con calidad alta de la evidencia. No parece tener efecto en la incidencia de mortalidad, evento cerebrovascular no fatal o enfermedad renal terminal [23].

De forma similar, dos revisiones sistemáticas publicadas compararon el tratamiento intensificado con hipoglucemiantes o insulina, con el fin de llegar a metas de $\mathrm{HbAc1}$ por debajo del $7 \%$ (TI) contra esquemas en los cuales el objetivo era obtener cifras de $\mathrm{HbA1c}$ del $7 \%$ o mayores, analizando de forma independiente los pacientes con antecedente de enfermedad coronaria [16,24]. La primera de ellas [16] documentó que cuando se comparaba el manejo intensivo con el manejo convencional en pacientes con antecedente de enfermedad cardiovascular en relación con aquellos que no la tenían no hubo cambio respecto al riesgo de morir por causa cardiovascular, con un RR de 1,13 (IC95\%: 0,81-1,57) vs. sin antecedente (RR: 0,89; IC95 \%: 0,74-1,08).

$\mathrm{Al}$ analizar de forma independiente el estudio ACCORD [25], donde un porcentaje significativo de pacientes tuvo antecedente de enfermedad cardiovascular establecida, encontró que el manejo intensivo comparado con el convencional no generaba cambio en el riesgo de sufrir el desenlace primario; por el contrario, el estudio fue terminado de forma temprana al observar mayor mortalidad en el grupo de manejo intensivo que en el convencional. En el análisis prestablecido de subgrupos no se encontró diferencia en la incidencia de mortalidad entre los que tenían y los que no tenían enfermedad cardiovascular previa, aunque estos últimos tuvieron una incidencia significativamente menor del desenlace compuesto primario.

Esto se demostró también en una segunda revisión sistemática de cuatro estudios que incluyó el anterior [24], donde el análisis exploratorio de subgrupos mostró que al comparar la terapia intensiva con la convencional, parecía haber un efecto diferencial en desenlaces cardiovasculares mayores en pacientes con antecedentes de enfermedad macrovascular y sin estos, siendo significativamente favorable para los últimos y no significativa para los primeros (HR: 1,00; IC95 \%: 0,89-1,13 vs. HR: 0,84; IC95 \%: 0,74-0,94, respectivamente; $\mathrm{p}=0,04$ para interacción). Por el contrario, en otro metanálisis los autores no encontraron interacción del antecedente de enfermedad isquémica del corazón con el efecto del control glucémico intensivo en eventos cardiovasculares, incluidos los fatales [26]. Tampoco se encontró heterogeneidad entre estudios con menos y con más del $20 \%$ de pacientes incluidos con enfermedad cardiovascular previa [16].

\section{Conclusión}

Teniendo en cuenta la información presentada, el panel consideró que no existe un beneficio significativo en iniciar tratamiento intensificado enfocado en obtener como meta de manejo una $\mathrm{HbA} 1 \mathrm{c}<7 \%$ en pacientes mayores de 65 años; por el contrario, este grupo de pacientes tiene un riesgo más alto de hipoglucemia, por lo que se decidió no recomendarlo.

Así mismo, el panel discutió que no existe evidencia alguna que demuestre que una meta de manejo más alta tenga beneficios clínicos, por lo que en pacientes mayores de 65 años sin otras comorbilidades y funcionalmente independientes se deberá buscar una meta de manejo igual a la utilizada para pacientes menores de esa edad, es 
decir, una $\mathrm{HbA} 1 \mathrm{c} \leq 7 \%$, tratando de minimizar el riesgo de hipoglucemia.

Deberán tenerse en cuenta subgrupos de pacientes mayores de 65 años, donde los riesgos de hipoglucemia son más altos, y, por tanto, requieren ajustar las metas de manejo. Si bien no existen experimentos clínicos que cuantifiquen ese riesgo, se decidió individualizar a los pacientes con fragilidad, demencia o depresión, así como a aquellos pacientes en cuidados de fin de vida. Para este enfoque se decidió tener en cuenta guías de práctica clínica desarrolladas por otros grupos y que generaron las recomendaciones de manejo basados en consenso de expertos [27,28,29]. Así mismo, se tuvieron en cuenta las definiciones de cuidados de fin de vida establecidas en la ley colombiana.

La decisión del grupo fue sugerir metas de manejo con un máximo de HbA1c del 8 $\%$, en pacientes funcionalmente dependientes, incluyendo aquellos con fragilidad o demencia, según las definiciones sugeridas por la Federación Internacional de Diabetes [28]. Adicionalmente, se decidió sugerir un objetivo terapéutico especial en pacientes en cuidados de fin de vida. Para ellos se sugiere como objetivo de manejo evitar que presenten síntomas asociados a hiperglucemia.

De acuerdo con la evidencia presentada, se consideró que la intensificación del manejo para llegar a metas de HbA1c menores del $7 \%$ en pacientes con antecedente de enfermedad cardiovascular no se asocia con reducción en el riesgo global de morir, ni en la incidencia de muerte de origen cardiovascular o de eventos cardiovasculares mayores. Respecto a eventos cerebrovasculares y enfermedad microvascular, no se dispone de datos específicos para este grupo de pacientes y, por ello, no es posible generar una recomendación basada en evidencia al respecto.

Se hace hincapié en que si bien la evidencia no demostró beneficio en intensificar el tratamiento para lograr concentraciones de $\mathrm{HbA} 1 \mathrm{c}$ menores al $7 \%$, tampoco hay evidencia para considerar una meta por encima de la establecida para la población sin eventos previos $(7 \%)$; por tanto, en pacientes con enfermedad cardiovascular se deben buscar las mismas metas que en la población general.

\section{Recomendaciones}

1. En los pacientes con DMT2 mayores de 65 años se recomienda no intensificar el manejo para alcanzar valores de $\mathrm{HbA1c}$ cercanos a lo normal (< 6,5\%). Recomendación fuerte en contra. Calidad de la evidencia: alta

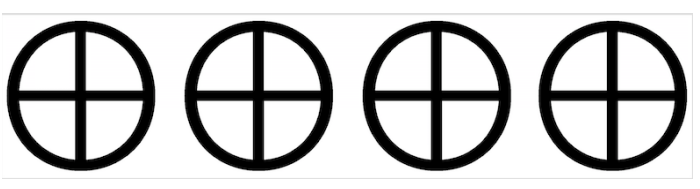

2. En los pacientes con DMT2 mayores de 65 años funcionalmente independientes y libres de otras comorbilidades mayores se recomienda dar manejo dirigido a alcanzar las mismas concentraciones de $\mathrm{HbA1c}$ recomendadas para la población menor de 65 años ( $\leq 7 \%$ ). Recomendación fuerte a favor. Calidad de la evidencia: alta

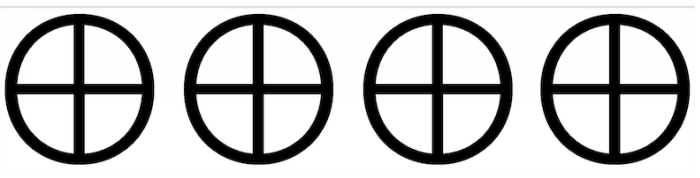

3. En los pacientes con DMT2 mayores de 65 años que presenten fragilidad ${ }^{1} \mathrm{o}$ demencia $^{2}$ o en quienes se anticipe riesgo aumentado de hipoglucemia ${ }^{3}$ se sugiere un manejo menos intensivo con valores de $\mathrm{HbA1c}$ entre el $7 \%$ y el $8 \%$. Recomendación débil a favor. Consenso de expertos

4. En pacientes con DMT2 que se encuentren en cuidados de fin de vida 4 se sugiere limitar el objetivo del tratamiento a evitar hiperglucemia

| Universitas Médica | V.58 | No.4 | 2017 | 
sintomática. Recomendación débil a favor. Consenso de expertos

5. En los pacientes con DMT2 y antecedente de enfermedad cardiovascular la meta de HbA1c no debe ser diferente a la meta general (igual o menor al $7 \%$ ). No se recomienda la intensificación del tratamiento para alcanzar un valor de $\mathrm{HbA1c}$ cercano al normal $(<6,5 \%)$.

\section{Punto de buena práctica clínica}

Considerando que la población mayor de 65 años es muy heterogénea, es necesario realizar una valoración funcional integral del paciente para establecer los objetivos terapéuticos.

\section{Referencias}

1. Aschner PJ, Ruiz AJ. Metabolic memory for vascular disease in diabetes. Diabetes Technol Ther. 2012 Jun;14 Suppl 1:S68-74.

2. Prospective Diabetes Study Gruup. Intensive blood-glucose control with sulphonylureas or insulin compared with conventional treatment and risk of complications in patients with type 2 diabetes (UKPDS 33). Lancet. 1998;352 (9131):837-53.

3. Effect of intensive blood-glucose control with metformin on complications in overweight patients with type 2 diabetes (UKPDS 34). UK Prospective Diabetes Study (UKPDS) Group. Lancet. 1998 Sep;352(9131):854-65.

4. Holman RR, Paul SK, Bethel MA, Matthews DR, Neil HAW. 10-year follow-up of intensive glucose control in type 2 diabetes. N Engl J Med. 2008 Oct;359(15):1577-89.

5. Gerstein HC, Miller ME, Byington RP, Goff DC, Bigger JT, Buse JB, et al. Effects of intensive glucose lowering in type 2 diabetes. N Engl J Med. 2008 Jun;358(24):2545-59.

6. Patel A, MacMahon S, Chalmers J, Neal B, Billot L, Woodward $\mathrm{M}$, et al. Intensive blood glucose control and vascular outcomes in patients with type 2 diabetes. N Engl J Med. 2008 Jun;358(24):2560-72.

7. Duckworth W, Abraira C, Moritz T, Reda D, Emanuele N, Reaven PD, et al. Glucose control and vascular complications in veterans with type 2 diabetes. N Engl J Med. 2009 Jan;360(2):129-39.

8. Inzucchi SE, Bergenstal RM, Buse JB, Diamant M, Ferrannini E, Nauck M, et al. Management of hyperglycaemia in type 2 diabetes: a patient-centered approach. Position statement of the American Diabetes Association (ADA) and the European Association for the Study of Diabetes (EASD). Diabetologia. 2012 Jun;55(6):1577-96.

9. Sinclair A, Paolisso G, Castro M, Bourdel I, Gadsby R, Rodriguez L. European Diabetes Working Party for Older People 2011 clinical guidelines for type 2 diabetes mellitus (EDWPOP). Diabetes Metab. 2011;37(3):s27-38.

10. Hewitt J, Smeeth L, Chaturvedi N, Bulpitt CJ, Fletcher a E. Self management and patient understanding of diabetes in the older person. Diabet Med. 2011 Jan;28(1):117-22.

11. Emerging Risk Factors Collaboration. Diabetes mellitus, fasting glucose, and risk of cause-specific death. N Engl J Med. 2011 Mar 3;364(9):829-41.

12. Rydén L, Grant PJ, Anker SD, Berne C, Cosentino F, Danchin N, et al. ESC guidelines on diabetes, pre-diabetes, and cardiovascular diseases developed in collaboration with the EASD. Eur Heart J. 2013;34(39):3035-87.

13. Inzucchi SE, Bergenstal RM, Buse JB, Diamant M, Ferrannini E, Nauck M, et al. Management of hyperglycemia in type 2 diabetes: a patient-centered approach: position statement of the American Diabetes Association (ADA) and the European Association for the Study of Diabetes (EASD). Diabetes Care. 2012;35(6):1364-79.

14. Riethof M, Pl F, Lindvall B, Michels R, Connor OP, Redmon B, et al. Diagnosis and 
Management of type 2 diabetes mellitus in adults. ICSI. 2012;(April).

15. Boussageon $R$, Bejan-Angoulvant $T$, Saadatian-Elahi M, Lafont S, Bergeonneau $\mathrm{C}$, Kassaï B, et al. Effect of intensive glucose lowering treatment on all cause mortality, cardiovascular death, and microvascular events in type 2 diabetes: meta-analysis of randomised controlled trials. BMJ. 2011 Jan;343:d4169.

16. Buehler AM, Cavalcanti AB, Berwanger O, Figueiro M, Laranjeira LN, Zazula AD, et al. Effect of tight blood glucose control versus conventional control in patients with type 2 diabetes mellitus: a systematic review with meta-analysis of randomized controlled trials. Cardiovasc Ther. 2013 Jun;31(3):147-60.

17. Coca S, Faramarz I, Nowreen H. Role of intensive glucose control in development of renal end points in type 2 diabetes mellitus. Arch Intern Med. 2012;172(10):761-9.

18. Hemmingsen B, Lund SS, Gluud C, Vaag $\mathrm{A}$, Almdal $\mathrm{T}$, Hemmingsen $\mathrm{C}$, et al. Intensive glycaemic control for patients with type 2 diabetes: systematic review with meta-analysis and trial sequential analysis of randomised clinical trials. BMJ. 2011 Jan;343(November):d6898.

19. Ray KK, Seshasai SRK, Wijesuriya S, Sivakumaran R, Nethercott S, Preiss D, et al. Effect of intensive control of glucose on cardiovascular outcomes and death in patients with diabetes mellitus: a meta-analysis of randomised controlled trials. Lancet. Elsevier Ltd; 2009 May 23;373(9677):1765-72.

20. Zhang C, Zhou Y-H, Xu C-L, Chi FL, Ju H-N. Efficacy of intensive control of glucose in stroke prevention: a metaanalysis of data from 59,197 participants in 9 randomized controlled trials. PLoS One. 2013 Jan;8(1):e54465.

21. Miller ME, Williamson J, Gerstein H. Effects of randomization to intensive glucose control on adverse events, cardiovascular disease and mortality in older versus younger adults in the ACCORD trial. Diabetes Care. 2014;37(3):634-43.

22. Anderson RT, Narayan KMV, Feeney P, Goff D, Ali MK, Simmons DL, et al. Effect of intensive glycemic lowering on health-related quality of life in type 2 diabetes: ACCORD trial. Diabetes Care. 2011 Apr;34(4):807-12.

23. Hemmingsen B, Lund S, Gluud C, Vaag A, Almdal T, Hemmingsen C, et al. Targeting intensive glycaemic control versus targeting conventional glycaemic control for type 2 diabetes mellitus. Cochrane Database Syst Rev. 2013;Nov 11;11:CD008143. doi: 10,1002/14651858.

24. Turnbull FM, Abraira C, Anderson RJ, Byington RP, Chalmers JP, Duckworth WC, et al. Intensive glucose control and macrovascular outcomes in type 2 diabetes. Diabetologia. 2009;52(11):2288-98.

25. Anderson RT, Narayan KMV, Feeney P. Effect of intensive glycemic lowering on healthrelated quality of life in. Diabetes Care. 2011;34:807-12.

26. Mannucci E, Monami M, Lamanna C, Gori F, Marchionni N. Prevention of cardiovascular disease through glycemic control in type 2 diabetes: A meta-analysis of randomized clinical trials. Nutr Metab Cardiovasc Dis. 2009;19(9):604-12.

27. European Diabetes Working Party for Older People working Group. European Diabetes Working Party for Older People 2011 Clinical Guidelines for Type 2 Diabetes Mellitus (EDWPOP). Diabetes Metab. 2011;37(3):S27-38.

28. Guideline Group. International Diabetes Federation. Managing older people with type 2 diabetes. 2004 [internet]. Disponible en: https://www.idf.org/sites/default/files/I DF\%20Guideline\%20for\%200lder\%20Pe ople.pdf

29. Gómez Huelgas R. Tratamiento de la diabetes tipo 2 en el paciente anciano. Med Clin. 2012;134(3):e1-134. 


\section{Notas}

1 Pacientes con fatiga significativa, restricción severa en la movilidad y fuerza, con riesgo incrementado de caídas y de institucionalización.

2 Pacientes con déficit cognitivo que condiciona problemas significativos de memoria, desorientación, cambios de personalidad o que tengan dificultades para autocuidado.

3 Pacientes en tratamiento con drogas que causan hipoglucemia (sulfonilureas, metiglinidas o insulina) y tienen otros factores que puedan incrementar este riesgo (depresión, pobre apoyo social, inapetencia o intolerancia a la ingesta).

4 Pacientes que cumplan con los criterios de enfermo en fase terminal definidos en la Ley colombiana 1733 de 2014 o la Resolución 1216 de 2015 (referente a derecho a morir con dignidad). Se define enfermo en fase terminal a todo aquel que es portador de una enfermedad o condición patológica grave, que haya sido diagnosticada de forma precisa por un médico experto, que demuestre un carácter progresivo e irreversible, con pronóstico fatal próximo o en plazo relativamente breve, que no sea susceptible de un tratamiento curativo y de eficacia comprobada, que permita modificar el pronóstico de muerte próxima; o cuando los recursos terapéuticos utilizados con fines curativos han dejado de ser eficaces. 